\title{
Avaliação objetiva do hipocratismo digital em imagens de sombra de dedo indicador; estudo em pacientes pneumopatas e em indivíduos normais*
}

\author{
Objective evaluation of clubbing on shadow images of index fingers. A study \\ of patients with pulmonary disease and of normal individuals.
}

\author{
JOSÉ DA SILVA MOREIRA, NELSON DA SILVA PORTO, ANA LUIZA SCHNEIDER MOREIRA
}

Introdução: 0 diagnóstico do hipocratismo digital é clínico, mas pode tornar-se mais acurado pelo uso de critérios objetivos de determinação.

Objetivo: Mostrar um método simples de obtenção de imagens de dedos para estudo do hipocratismo digital.

Método: Em imagens de dedos indicadores em perfil, obtidas praticamente sem distorção em folha de papel comum, projetadas através de lâmina de vidro, determinaram-se os ângulos do perfil e hiponiquial, e a relação entre as espessuras falangeana distal e interfalangena. Estudaram-se 306 pneumopatas adultos, clinicamente com hipocratismo presente, ausente ou duvidoso, e 452 indivíduos adultos normais. Eram fumantes $71,0 \%$ dos pacientes e $33,4 \%$ dos controles.

Resultados: 0s valores encontrados nos indivíduos normais e nos pacientes nos quais havia a presença clínica de hipocratismo foram, respectivamente, $172,8 \pm 5,3^{\circ}$ e $183,4 \pm$ $5,0^{\circ}$ para ângulo de perfil, $181,5 \pm 4,8^{\circ}$ e $201,4 \pm 6,5^{\circ}$ para ângulo hiponiquial, e 0,904 $\pm 0,029$ e 1,014 $\pm 0,062$ para a relação entre as espessuras falangeana distal e interfalangeana. As diferenças foram significativas. Os casos duvidosos também tiveram valores maiores que os verificados nos controles. No grupo controle, observou-se que os fumantes masculinos apresentaram os três valores significativamente superiores aos exibidos pelos não fumantes, enquanto que nas mulheres fumantes desse grupo apenas a relação entre as espessuras falangeana distal e interfalangeana se mostrou superior.

Conclusão: Imagens nítidas de dedos indicadores foram facilmente obtidas pelo método empregado. 0 ângulo hiponiquial, determinado a partir das imagens, foi a medida que se mostrou com maior capacidade para discriminar casos com e sem hipocratismo (sensibilidade de 76,7\%, especificidade de 83,2\%, valores preditivos positivo de 95,8\% e negativo de 96,9\%).

J Bras Pneumol 2004; 30(2) 36-44
Background: Normal diagnosis of clubbing is clinical; however use of objective criteria may improve the accuracy of findings

Objective: To present a simple method of obtaining finger images for the purpose of studying clubbing.

Method: Shadow images of the index fingers obtained by projection through a transparent glass plate virtually without distortion and displayed on a common sheet of paper yielded the profile (PA) and hyponychial ( $\mathrm{HA})$ angles; as well as the ratio between distal phalangean and interphalangean depths (DPD/IPD). Upon physical examination of 306 adult bearers of pulmonary disease, 116 disclosed presence of clubbing (YES); 126 absence (NO); and 64 were doubtful cases (DBT). Also studied were 452 normal adult individuals. Among these $71.0 \%$ of the bearers and $33.4 \%$ of the controls were smokers.

Results: Values found in normal individuals and in patients bearers of clubbing (YES) were, respectively, $172.8 \pm 5.9^{\circ} \mathrm{vs}$. $183.4 \pm 5.9^{\circ}$ for PA, $181.5 \pm 5.0^{\circ}$ vs. $201.4 \pm 6.5^{\circ}$ for HA, and $0.904 \pm 0.029$ vs. $1.014 \pm 0.062$ for DPD/IPD (significant differences, $\mathrm{p}<0.001)$. In the doubtful) cases (DBT) the three values were also higher than in normal controls $(p<0.001)$. Furthermore, it was shown that among controls PA, HA and the DPD/IPD ratios were significantly larger in male smokers $(p<0.005)$ while only the DPD/IPD ratio was larger in female smokers $(\mathrm{p}<0.05)$.

Conclusions: This is a simple method of obtaining clear index finger images. The hyponychial angle determined on the images was the most useful measurement to discriminate digits clinically with and without clubbing (sensitivity of $76.7 \%$, specificity of $83.2 \%$, predictive positive value of $95.5 \%$ and predictive negative value of 96.9\%).

Key words: Clubbing, profile angle, hyponychial angle, relation $\mathrm{DPD} / \mathrm{IPD}$.
Descritores: Hipocratismo digital, baqueteamento, ângulo do perfil, ângulo hiponiquial, relação entre espessuras do dedo. 


\section{Veja Editorial, na página 92}

\section{INTRODUÇÃO}

0 hipocratismo digital é um valioso sinal clínico indicador de doença intratorácica pulmonar ou cardíaca $^{(1,2)}$, podendo algumas vezes, todavia, estar relacionado a hepatopatias crônicas ${ }^{(3)}$, doenças do intestino ${ }^{(4)}$, da tireóide ${ }^{(5)}$ ou ainda ser hereditário(6). É reconhecido desde a época de Hipócrates ${ }^{(7)}$, e por muito tempo considerou-se ser relacionado com a tuberculose, embora Pigeaux, em 1832, já tivesse atribuído seu aparecimento a uma gama de situações mais ampla, como um "vício na hematose" ${ }^{(8)}$. Ele pode apresentar-se isolado ou encontrar-se incluido na sindrome de osteoartropatia hipertrófica. As naturezas tanto do hipocratismo como dessa síndrome, entretanto, ainda não estão esclarecidas. Apesar de terem sido formuladas algumas teorias para explicá-las ${ }^{(9,10)}$, nenhuma delas logrou êxito até o momento. Estudos recentes admitem que citocinas, que atuam como fatores de crescimento, ligadas a megacariócitos ou não, podem se encontrar envolvidas na patogenia das anormalidades ${ }^{(11,12)}$.

0 hipocratismo não oferece dificuldades em seu diagnóstico naqueles casos cuja presença é clinicamente óbvia, com alterações grosseiras nas extremidades dos dedos, mesmo que se usem diferentes critérios clínicos para a sua detecçã $0^{(13)}$, e variada sinonímia para sua designaçã $0^{(14)}$. Todavia, o diagnóstico pode não ser tão fácil quando as modificações são incipientes, pouco pronunciadas $^{(15,16)}$. Nestes casos, especialmente, critérios objetivos de determinação mostram-se vantajosos, auxiliam o juízo clínico, e ainda possibilitam que os dados fiquem registrados e armazenados para estudos posteriores.

Dentre os critérios de avaliação objetiva do hipocratismo digital, os que têm se mostrado mais fidedignos são as medidas do ângulo ou sinal do perfil verificado em dedos indicadores $(\mathrm{Pi})^{(17)}$ ou polegares ${ }^{(18)}$, do ângulo hiponiquial em dedos indicadores $(\mathrm{Hi})^{(19)}$, e a relação entre as espessuras falangeana distal e interfalangeana de dedos indicadores (EFD/EIF) ${ }^{(20,21)}$. Para que esses critérios possam ser aplicados, entretanto, faz-se necessário que se obtenham imagens em perfil dos $\operatorname{dedos}^{(22)}$, ou moldes rígidos dos mesmos ${ }^{(23)}$. Determinações do grau de curvatura da unha ${ }^{(24,25)}$, estimativas de aumento do volume da extremidade do dedo ${ }^{(26)}$, radiogramas ${ }^{(27)}$ e terrmografias ${ }^{(28)}$ têm sido também explorados na avaliação do hipocratismo e da osteoartropatia
Siglas e abreviaturas utilizadas neste trabalho:

DP - Desvio-padrão

DUV -Dúvida quanto à presença clínica de hipocratismo digital

EFD - Espessura falangiana distal

EIF - Espessura interfalangiana

$\mathrm{Hi}$ - Ângulo hiponiquial do dedo indicador

NÃO - Ausência clínica de hipocratismo digital

Pi - Ângulo de perfil do dedo indicador

SIM - Presença clínica de hipocratismo digital.

hipertrófica. Todos esses métodos procuram traduzir o aumento dos tecidos moles que ocorre nos locais envolvidos, especialmente nas regiões sub-ungueais das extremidades dos dedos ${ }^{(29-31)}$.

Alguns valores referidos na literatura, para os critérios utilizados neste estudo, em indivíduos normais, são: $168,3^{\circ} \pm 3,6^{o(32)}$ e $171,4^{\circ} \pm 5,5^{\circ}$ para o $\mathrm{Pi} ; 186,0^{\circ} \pm 2,0^{\circ}{ }^{(19)}, 180,1^{\circ} \pm 4,2^{\circ}{ }^{(32)} \mathrm{e}$ $180,7^{\circ} \pm 5,2^{\circ(33)}$ para o $\mathrm{Hi}$; e $0,895 \pm 0,041 \mathrm{em}$ crianças ${ }^{(16)}, 0,889 \pm 0,037$ em adultos da raça negra e 0,911 $\pm 0,049$ em caucasianos adultos, para a relação EFD/ElF ${ }^{(34)}$. Em individuos clinicamente portadores de hipocratismo, todos esses valores apresentam-se significativamente maiores, mostrando-se bons discriminadores de dedos com e sem anormalidade.

0 objetivo do presente trabalho é mostrar um modo simples e rápido de obtenção de imagens em perfil de dedos indicadores, sobre as quais se pode usar critérios objetivos, já conhecidos, de avaliação do hipocratismo digital.

\section{MÉTODO}

Para a obtenção das imagens, projetava-se a sombra do perfil do dedo indicador através de uma lâmina de vidro plano, e recolhia-se a imagem em uma folha de papel branco comum, colocada sobre o vidro. 0 dedo indicador a ser examinado, em perfil correto, ficava junto e por debaixo da lâmina de vidro, e a fonte luminosa verticalmente a cerca de $70 \mathrm{~cm}$ mais abaixo. A fonte pode ser uma lâmpada elétrica incandescente comum ou uma lanterna simples.

Na prática, a lâmina de vidro ficava sobre uma mesa e a lâmpada no chão. A Figura 1 ilustra a montagem do processo de obtenção da imagem. 0 dorso da mão deve encostar na mesa e o dedo polegar no vidro, a fim de prevenir deslocamentos. 
Sobre a imagem obtida da sombra do dedo colocavam-se as demarcações para as determinações do $\mathrm{Hi}$, do $\mathrm{Pi}$ e da relação EFD/EIF. Elas eram colocadas nos seguintes pontos: prega cutânea dorsal distal; epiniquio; ponto mais elevado da superfície dorsal da unha; hiponíquio; ponto ventral, diametralmente oposto ao epiníquio; e ponto ventral, diametralmente oposto àquele referente à prega cutânea interfalangeana dorsal distal (Figura 2). Na Figura 3 observam-se o aspecto definitivo do conjunto de pontos de demarcação sobre o papel, agora já sem a presença da sombra do dedo, e os ângulos e distâncias traçados pelos referidos pontos. Na quase totalidade das vezes escolheu-se o dedo indicador da mão direita, usando-se o indicador da mão esquerda somente em presença de lesão ou na falta do dedo direito (em geral por trauma). Para as demarcações utilizaram-se lápis de ponta afiada, régua com calibração de meio milimetro e transferidor de $360^{\circ}$ com calibração de meio grau. Todas as leituras, tanto nos pacientes como nos individuos normais, foram efetuadas pelo mesmo pesquisador. A reprodutibilidade do método foi inicialmente testada repetindo-se por seis vezes as determinações de $\mathrm{Pi}$, $\mathrm{Hi}$ e da relação EFD/EIF, no mesmo dedo indicador de doze individuos normais.

0 método foi aplicado em 306 pacientes (241 homens e 65 mulheres), com idades entre 15 e 88 anos (média de 51,3 anos), portadores de doenças pulmonares diversas e em 452 individuos adultos normais e sadios (233 homens e 219 mulheres), com idades entre 15 e 80 anos (média de 40,2 anos). Os pacientes foram todos selecionados em um hospital universitário, especializado em doenças pulmonares.

Entre os 306 pacientes, internados e investigados, as doenças mais freqüentemente diagnosticadas foram carcinoma brônquico, doença pulmonar obstrutiva crônica, bronquiectasias e fibrose pulmonar. Eram fumantes 217 (71\%) deles. Em 116 pacientes o exame clínico concluiu pela presença (SIM) de hipocratismo, em 126 por sua ausência (NÃo), e em 64 havia dúvida (DUV) quanto à presença do sinal.

Os individuos componentes do grupo controle eram inteiramente assintomáticos, nenhum deles apresentava clinicamente

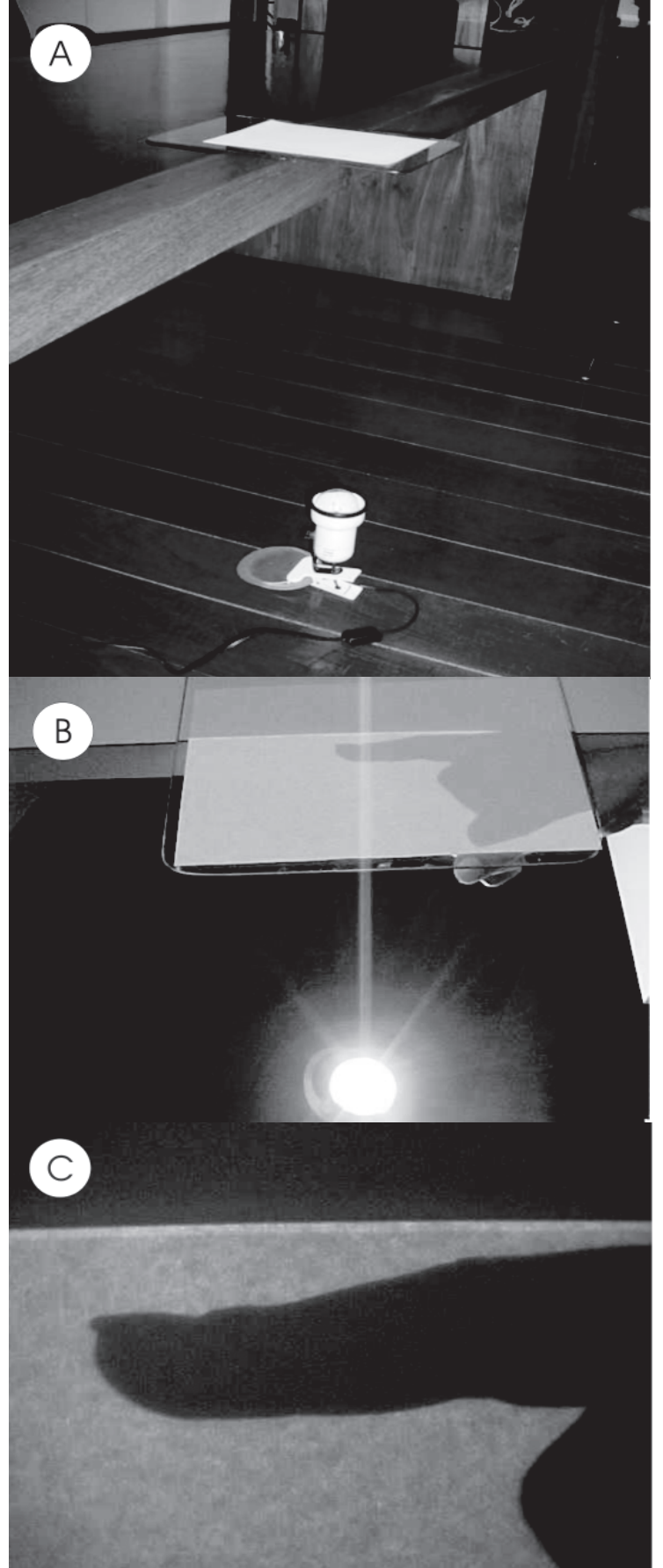

Figura 1. Esquema montado para a obtenção da imagem da sombra do dedo. a) Folha de papel comum sobre lâmina de vidro plano, a qual se encontra sobre a mesa. Lâmpada comum colocada no chão, verticalmente abaixo. b) Os dedos indicador e polegar ficam sob a lâmina de vidro, encostados nela, e o dorso da mão encostado na mesa. c) A folha de papel, sobre o vidro, recolhe a imagem de perfil do dedo indicador. 
hipocratismo digital, e 301 (66,6\%) deles nunca haviam fumado, o que constituiu diferença significativa ( $p<0.001)$ com relação ao grupo de estudo (pacientes). Havia 151 fumantes (por mais de cinco anos) nesse grupo, dos quais 82 eram homens e 69 mulheres. Os homens fumavam 15,7 $\pm 7,2$ cigarros ao dia e a mulheres $13,0 \pm 7,2$, uma diferença significativa ( $p<$ 0,05). Pertenciam à raça branca 354 (78,3\%), e à raça negra $98(21,7 \%)$ deles. A atividade manual, em especial das extremidades dos dedos, foi considerada leve em 164 (36,3\%) desses indivíduos, moderada em 220 (48,7\%) e pesada em 68 (15,0\%), grupo este formado por cirurgiões, pianistas, organistas, datilógrafos e digitadores. A atividade manual foi considerada leve em médicos não cirurgiões, estudantes e enfermeiros, e moderada em trabalhadores em geral, sem uso específico das extremidades dos dedos. A maioria dos controles, 385 (85,2\%), referiu ter um radiograma convencional de tórax normal, e 341 destes, efetuado o radiograma dentro dos últimos cinco anos.

0 exame dos dedos dos pacientes e dos controles foi efetuado por médicos experientes, treinados em doenças pulmonares, os quais forneceram sua impressão clínica quanto à presença do hipocratismo. Nos casos duvidosos, o clínico deveria optar pela maior tendência, positiva ou negativa. Os dados objetivos das mensurações foram contrapostos à impressão subjetiva quanto à presença do sinal clínico, de modo semelhante ao que foi feito por Regan et al. ( 19).

0 consentimento informado foi obtido de todos os pacientes e indivíduos do grupo controle que participaram do estudo.

Testes de média foram empregados (testes $\mathrm{t}$ ) para a comparação dos valores numéricos encontrados, e testes do Qui-quadrado para dados que envolveram valores proporcionais. Adotou-se o nível de significância de 5\%. Sensibilidade, especificidade e valor preditivo positivo foram determinados para os ângulos e relação entre espessuras, tendo-se a impressão clínica como padrão para comparação. Para isto, os 64 casos considerados clinicamente como duvidosos foram distribuídos entre NÃO (33 casos) e SIM (31 casos), de acordo com a direção da tendência seguida pela conclusão clínica.
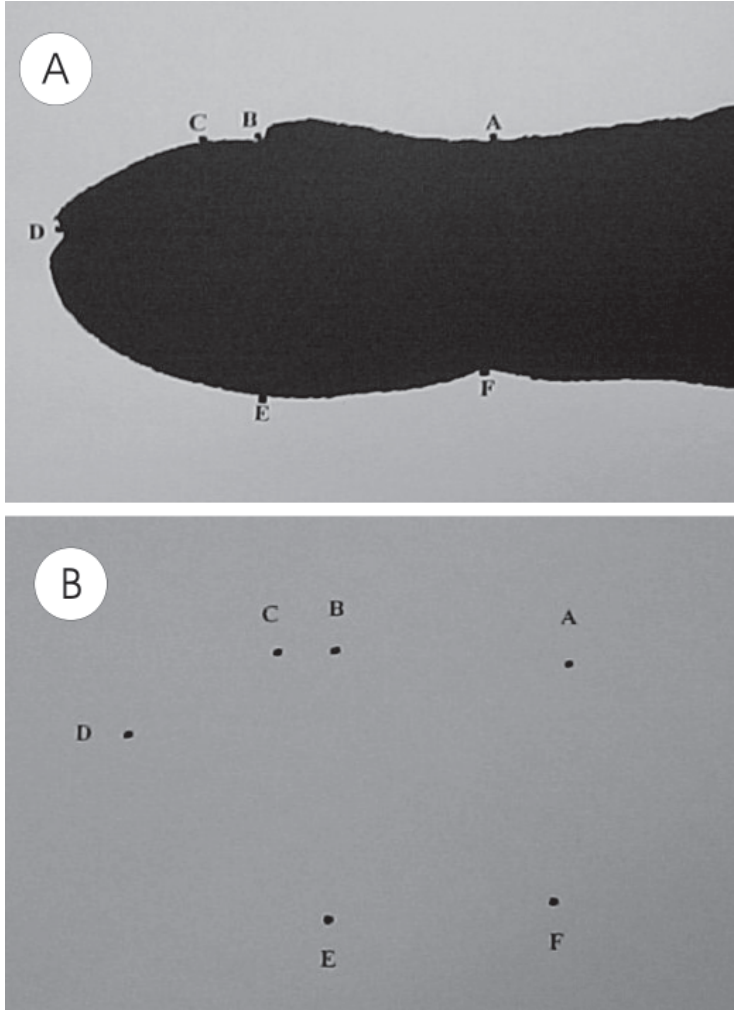

Figura 2. (a) Demarcações no papel colocadas sobre a imagem da sombra do dedo: A) prega cutânea distal dorsal, B) epiníquio, C) porção mais elevada da superfície da unha, D) hiponíquio, E) ponto ventral diametralmente oposto ao epiníquio, F) ponto ventral diametralmente oposto à prega cutânea distal dorsal. (b) Pontos demarcados, já sem a sombra do dedo.

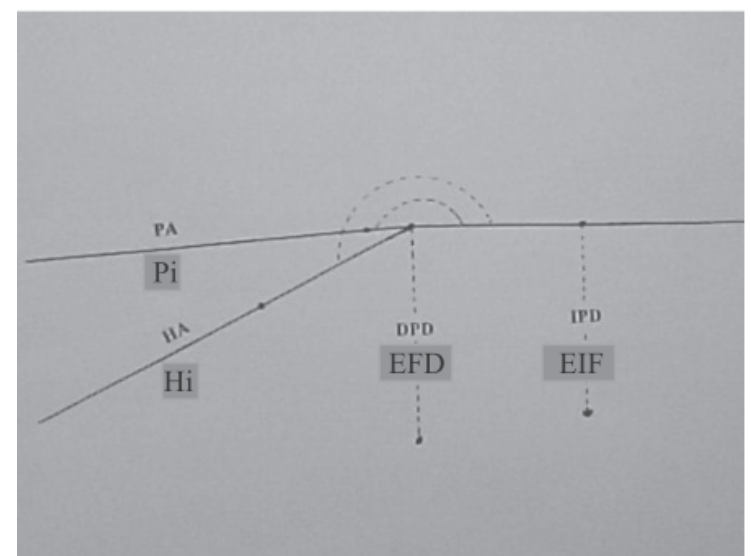

Figura 3. Conjunto dos pontos demarcados, sem a presença da sombra do dedo, com determinação dos ângulos do perfil ( $\mathrm{Pi})$, hiponiquial $(\mathrm{Hi})$ e das espessuras EFD e EIF. 


\section{RESULTADOS}

$\mathrm{Na}$ avaliação da reprodutibilidade do método, através do coeficiente de variação, verificou-se variabilidade inferior a $2,5 \%$ nas repetidas determinações efetuadas de $\mathrm{Pi}$, Hi e relação EFD/ EIF em um mesmo dedo, e observou-se a menor delas na relação EFD/EIF (Tabela 1).

As análises dos valores de $\mathrm{Pi}, \mathrm{Hi}$ e da relação EFD/EIF, verificados nos dedos indicadores dos 452 indivíduos do grupo controle, são mostradas na Tabela 2. Esses valores, verificados nos dedos dos indivíduos do grupo controle, mostraram-se independentes em relação ao sexo, idade, raça e atividade manual efetuada pelos componentes desse grupo. Entretanto, os valores de $\mathrm{Pi}$ e Hi e da relação entre EFD/EIF em 82 homens fumantes do mesmo grupo foram significativamente maiores $(p<0,005)$ que os encontrados nos não fumantes (Tabela 3). Nas 69 mulheres fumantes somente o valor da relação EFD/EIF mostrou-se levemente superior $(0,911 \pm 0,029)$ ao verificado nas 150 não fumantes $(0.902 \pm 0,030)$ de modo significativo $(p=0,037)$.

$\mathrm{Na}$ Tabela 4 são reunidos os valores encontrados para $\mathrm{Pi}$, Hi e para a relação EFD/ EIF nos 306 pacientes pneumopatas estudados, clinicamente considerados não portadores de hipocratismo digital, com a presença do sinal duvidosa e com hipocratismo, e nos 452 indivíduos do grupo controle. Os pacientes com hipocratismo e aqueles em que a presença do sinal foi duvidosa tiveram os três valores significativamente diferentes dos verificados nos pacientes sem hipocratismo ( $p<0,001)$. Os pacientes pneumopatas, considerados clinicamente sem hipocratismo, tiveram os valores do $\mathrm{Hi}$ e da relação EFD/ElF também maiores que nos indivíduos do grupo controle $(p<0.05)$.

Considerando-se como pontos de corte os valores médios encontrados para os ângulos e para a relação entre espessuras nos casos clinicamente duvidosos (DUV), e colocando-se os casos duvidosos cuja tendência era para a presença clínica do hipocratismo em "SIM" (31 casos), e aqueles com tendência para a ausência em "NÃO" (33 casos), determinaram-se sensibilidade, especificidade e valores preditivos positivo e negativo de $\mathrm{Pi}$, Hi e da relação EFD/EIF dos 306 pacientes (Tabela 5)

0 critério objetivo que melhor discriminou dedos com e sem hipocratismo foi o $\mathrm{Hi}$, com ponto de corte em 191,50 (Figura 4).

TABELA 1

Coeficiente de variação (CV) dos três valores Pi, Hi e EFD/EIF em 6 determinações no mesmo dedo indicador de cada um de 12 indivíduos (72 mensurações).

\begin{tabular}{cccc}
\hline & $\mathrm{Pi}$ & $\mathrm{Hi}$ & $\mathrm{EFD} / \mathrm{EIF}$ \\
\hline $\mathrm{CV}$ & $1,46 \%$ & $0,78 \%$ & $0,68 \%$ \\
& $(0,89-2,20 \%)$ & $(0,76-1,47 \%)$ & $(0,02-1,00 \%)$ \\
\hline
\end{tabular}

Pi: Ângulo do perfil, Hi: ângulo hiponiquial, EFD/EIF: relação entre as espessuras falangeana distal e interfalangeana.

TABELA 2

Valores dos ângulos do perfil (Pi) e hiponiquial (Hi) e da relação EFD/ElF verificados nos 452 indivíduos normais do grupo controle

\begin{tabular}{cccc}
\hline & Média & DP & IC $_{95_{0}}$ \\
\hline $\mathrm{Pi}$ & $172,8^{\circ}\left(161,5-187,0^{\circ}\right)$ & $5,3^{\circ}$ & $\left(172,5-173,5^{\circ}\right)$ \\
$\mathrm{Hi}$ & $181,5^{\circ}\left(171,0-194,0^{\circ}\right)$ & $4,8^{\circ}$ & $\left(181.3-181,7^{\circ}\right)$ \\
EFD/EIF & $0,904(0,862-0,960)$ & 0,029 & $(0,902-0,905)$ \\
\hline
\end{tabular}


TABELA 3

Valores dos ângulos do perfil Pi e hiponiquial (Hi) e da relação EFD/EIF em indivíduos normais (controles) do sexo masculino, não-fumantes e fumantes.

\begin{tabular}{cccc}
\hline & Não fumantes (151) & Fumantes (82) & $\mathrm{P}$ \\
\hline $\mathrm{Pi}$ & $172,5 \pm 5,3^{\circ}$ & $174,7 \pm 5,3$ & 0,003 \\
$\mathrm{Hi}$ & $179,8 \pm 5,5^{\circ}$ & $183,1 \pm 5,4^{\circ}$ & $<0,001$ \\
EFD/EIF & $0,897 \pm 0,028$ & $0,910 \pm 0,026$ & $<0,001$ \\
\hline
\end{tabular}

TABELA 4

Valores dos ângulos do perfil (Pi) e hiponiquial (Hi) , e da relação EFD/ElF nos 306 pacientes pneumopatas estudados, clinicamente sem (NÃO), duvidoso (DUV) e com (SIM) hipocratismo, e nos 452 controles normais.

\begin{tabular}{ccccc}
\hline & NÃO(126) & DUV(64) & $\operatorname{SIM}(116)$ & CONTROLES(452) \\
\hline $\mathrm{Pi}$ & $173,7 \pm 5,3^{\circ}$ & $178,8 \pm 4,0^{\circ}$ & $183,4 \pm 5,9^{\circ}$ & $172,8 \pm 5,3^{\circ}$ \\
& $(166,0-184,0)$ & $(175,0-186,0)$ & $(175,0-195,5)$ & $(161,5-187,0)$ \\
$\mathrm{Hi}$ & $184,3 \pm 4,5^{\circ}$ & $191,5 \pm 2,1^{\circ}$ & $201,4 \pm 6,5^{\circ}$ & $181,5 \pm 4,8^{\circ}$ \\
& $(174,0-193,0)$ & $\left(189,0-195,0^{\circ}\right)$ & $\left(190,5-222,0^{\circ}\right)$ & $\left(171,0-194,0^{\circ}\right)$ \\
EFD/EID & $0,917 \pm 0,036$ & $0,960 \pm 0,03$ & $1,014 \pm 0.062$ & $0,904 \pm 0,029$ \\
& $(0,853-0,967)$ & $(0,866-1,003)$ & $(0,917-1,176)$ & $(0,862-0,960)$ \\
\hline
\end{tabular}

Os valores de Pi, Hi e EFD/EIF verificados em SIM e em DUV foram significativamente maiores que os encontrados em NÃO e nos controles normais $\mathrm{p}<0,001)$. Os valores de Hi e EFD/EIF registrados em (NÃO) mostraram-se superiores aos dos controles normais ( $p<0.05)$.

\section{DISCUSSÃO}

A imagem da sombra do dedo no estudo do hipocratismo já havia sido explorada por Bentley e Cline em $1970^{(22)}$ e por Bentley et al. em $1976^{(32)}$, os quais usaram um dispositivo que projetava em um anteparo a sombra ampliada, construindo o desenho da sombra pelo traçado do perfil da extremidade do dedo. Siniah e Omar, em 1979, utilizaram sombras de dedos em perfil, obtidas através de retroprojetor, também recolhidas em anteparo ${ }^{(33)}$, com o que conseguiram boas imagens para análise do hipocratismo. 0 método aqui apresentado, entretanto, obtém as imagens da sombra dos dedos, e em especial as recolhe, de maneira diferente daquelas propostas nos trabalhos desses autores.

0 método descrito no presente trabalho é simples, não requer nenhum dispositivo especial, e pode ser rapidamente aplicável a um grande número de indivíduos, mesmo em seus próprios locais de trabalho ou residência. As imagens das sombras são nítidas, praticamente não sofrendo distorções projetivas, uma vez que o dedo fica junto à imagem formada e distante do foco luminoso. Os pontos de referência para o cálculo dos ângulos e espessuras são facilmente colocados sobre as imagens obtidas, e os valores mostramse reprodutíveis. Os registros colhidos em folhas de papel podem ser armazenados, com os dados objetivos mensurados, com mínima ocupação de espaço, permanecendo especialmente úteis para estudos comparativos referentes à evolução do sinal clínico, particularmente na documentação da regressão da alteração após o tratamento da doença básica.

Os valores encontrados para $\mathrm{Pi}$, $\mathrm{Hi}$ e para a relação EFD/EIF nos indivíduos normais (controles) concordam com o que tem sido registrado na literatura com o emprego de outros métodos $^{(16,21,33,34)}$. 0 número de indivíduos sadios estudados, cuidadosamente selecionados, a maioria deles não fumantes, e com um radiograma de tórax referido como normal, asseguram consistência aos valores, os quais foram 
TABELA 5

Sensibilidade (SENS), especificidade (ESPEC) e valores preditivos positivo (VPP) e negativo (VPN) de Pi, Hi e EFD/EIF verificados nos 306 pacientes pneumopatas.

\begin{tabular}{ccccc}
\hline & SENS (\%) & ESPEC (\%) & VPP (\%) & VPN (\%) \\
\hline $\mathrm{Pi}$ & 70,0 & 71,7 & 88,7 & 84,8 \\
$\mathrm{Hi}$ & 76,7 & 85,0 & 95,8 & 96,9 \\
EFD/EIF & 70,0 & 74,2 & 88,6 & 84,9 \\
\hline
\end{tabular}

Pi: ângulo do perfil Hi: ângulo hiponiquial EFD/ElF: relação entre as espessuras da extremidade dos dedos indicadores.

significativamente menores que os verificados no grupo dos pacientes, especialmente naqueles com hipocratismo digital clinicamente presente ou duvidoso.

0 fato de o Hi mostrar-se com os valores mais elevados de sensibilidade, especificidade e valores preditivos positivo e negativo, na discriminação de pacientes com e sem hipocratismo digital devese, provavelmente, à capacidade que esta medida tem de considerar ao mesmo tempo o aumento da espessura dos tecidos moles sub-ungueais e o grau de curvatura da unha ${ }^{(19)}$. A faixa da dúvida clínica quanto à presença de hipocratismo, no grupo dos doentes situou-se entre os valores $189,0^{\circ}$ e $195,0^{\circ}$. Entretanto, é bem provável que a maioria dos casos com valores do $\mathrm{Hi}$ superiores a $191,5^{\circ}$ (ponto de corte) tenham clinicamente hipocratismo digital, especialmente se forem considerados os resultados encontrados nos controles, nos quais os valores foram significativamente mais baixos. A sensibilidade, a especificidade e os valores preditivos encontrados com a metodologia empregada permitem sugerir que o $\mathrm{Hi}$, especialmente, até o momento, seja o mais apropriado critério objetivo para conferir o diagnóstico clínico do hipocratismo digital.

Foi visto, também, que nos indivíduos do grupo controle fumantes do sexo masculino, os três valores analisados, e nas mulheres a relação entre as espessuras, mostraram-se maiores que os encontrados nos não fumantes, sugerindo que as alterações pulmonares determinadas pelo hábito tabágico, que foi mais intenso nos homens, podem modificar as extremidades dos
ESPECIFICIDADE (\%)

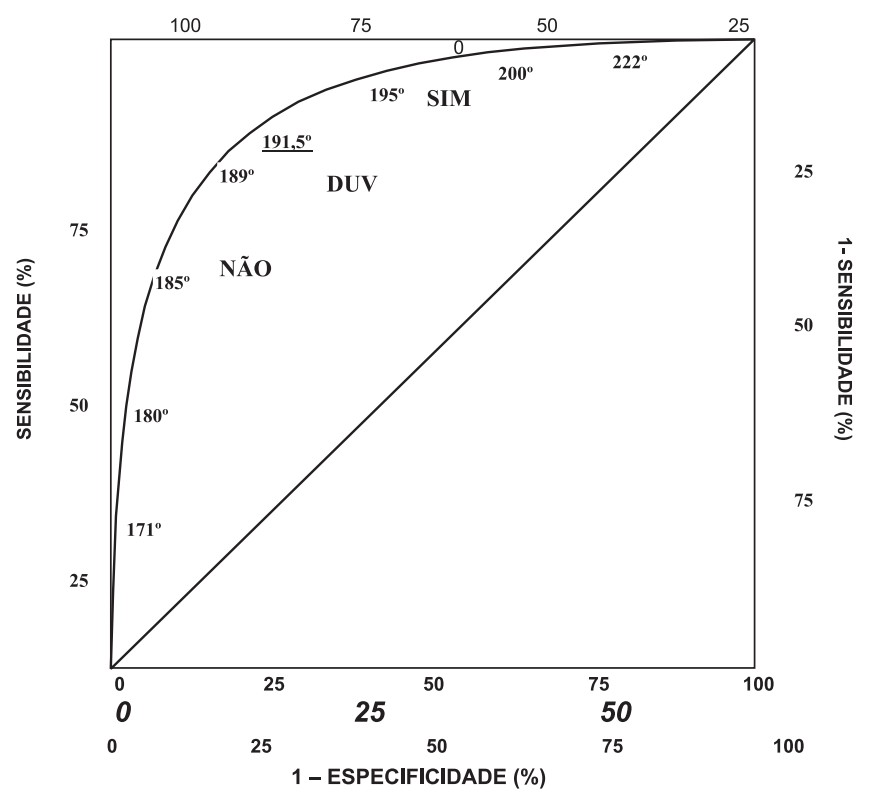

Figura 4. Curva ROC (Receiver Operator Characteristic curve) para o diagnóstico do hipocratismo digital de acordo com os valores do ângulo hiponiquial $(\mathrm{Hi})$, contrapostos à impressão clínica. A faixa da dúvida clínica quanto à presença do sinal fica entre $189,0^{\circ}$ e $195,0^{\circ}$. Ponto de corte em $191,5^{\circ}$.

dedos, ainda que em nível sub-clínico. É possível que alterações nas pequenas vias aéreas descritas em indivíduos fumantes ${ }^{\left({ }^{5}\right)}$ possam ter algum papel em relação a esse achado. Mais recentemente foram descritos, em material de biópsia pulmonar, casos de indivíduos adultos do sexo masculino com doença intersticial 
pulmonar associada à bronquiolite (RBLID "respiratory bronchiolitis-associated instertitial lung disease"), em que havia severa dispnéia, hipoxemia ao exercício e hipocratismo digital, e cujos sinais, sintomas e provas funcionais respiratórias melhoraram significativamente poucos meses após a interrupção do uso do fumo (36). No presente trabalho, a detecção dessas pequenas diferenças de valores das mensurações observadas entre indivíduos normais fumantes e não fumantes pode ter sido possibilitada pelo número elevado de indivíduos estudados.

No presente estudo, também se verificou que os valores do ângulo hiponiquial e da relação entre as espessuras do dedo, verificados nos pacientes considerados clinicamente não portadores de hipocratismo, se mostraram significativamente maiores que nos indivíduos do grupo controle. Eles, todavia, eram todos portadores de alguma doença pulmonar, além de serem também fumantes em sua maioria. Em 1972, Sly et al. ${ }^{(16)}$ já haviam referido que a relação EFD/EIF, determinada objetivamente em moldes rígidos de dedos indicadores, se mostrara maior em crianças asmáticas que em sadias, o que eles designaram mínimo hipocratismo. Goyal et al. (15) referiram que o hipocratismo poderia ser precocemente detectado por determinações objetivas, como pela análise morfométrica das unhas.

\section{REFERÊNCIAS}

1. Dickinson CJ. Lung diseases associated with digital clubbing. Clin Exp Rheumatol 1992;10(Suppl 7):23-5.

2. Moreira JS, Rubin AS, Silva LCC, Silva FAA, Hetzel JL. Clubbing: frequency in several pulmonary diseases. Eur Respir J 2000; 16(Suppl 31):422.

3. Stoller JK. As the liver does, so goes the lung. Chest 1990;97:1028-30.

4. Fielding JF, Cooke WT. Finger clubbing and regional enteritis. Gut 1971;123:442-4.

5. Gotte D. Thyroid acropachy. Arch Dermatol 1980;116:201-8.

6. Fisher DS, Singer DH, Feldman SM. Clubbing, a review with emphasis on hereditary acropachy. Medicine 1964;43:459-79.

7. Hippocrates: the book of prognostics. In: Major RH. Classic descriptions of disease. 3rd ed. Springfield: Charles C Thomas; 1945. p.4-5.

8. Pigeaux J. Recherches nouvelles sur l'étiologie, la symptomatologie et le mécanisme du developpement fusiforme de l'extremité des doigts. Arch Gen Med 1832;29:174-84.

9. Martinez-Lavin M. Digital clubbing and hyperthrophic osteoarthropathy: a unifying hypothesis. J Rheumatol 1987; 14:6-8.

10. Shneerson JM. Digital clubbing and hypertrophic osteoartropathy: The underlying mechanisms. $\mathrm{Br} \mathrm{J}$ Dis Chest 1981;75:113-29.
11. Silveri F, De Angelis R, Argenmtati F, Muti S, Cervini C. Hypertrophic osteoathropathy: endothelium and platelet function. Clin Rheumatol 1996;15:435-9.

12. Yosipovitch G, Weinberger A. Cytokines. A unifying concept in pathogenesis of clubbing. Med Hypotheses 1991;36:122-5.

13. Myers KA, Farquhar DRE. Does this patient have clubbing? JAMA $2001 ; 282: 341-7$.

14. Mendlowitz M. Clubbing and hypertrophic osteoarthropathy. Medicine 1942;21:269-306.

15. Goyal S, Griffiths AD, Omarouayache S, Mohammedi R. An inproved method of studying fingernail morphometry: application to the early detection of fingernail clubbing. J Am Acad Dermatol 1998;39:640-2.

16. Sly RM, Fuqua G, Matta G, Waring WW. Objective assessment of minimal digital clubbing in asthmatic children. Ann Allergy 1972;30:575-8.

17. Trousseau A. Forme hipocratique des doigts des tuberculeux. J Conaiss Med Cir 1834;1:351-2.

18. Lovibond JL. Diagnosis of clubbed fingers. Lancet 1938;1:363-4.

19. Regan BM, Tagg B, Thomson ML. Subjective assessment and objective measurement of finger clubbing. Lancet 1967;1:530-2.

20. Rice RE, Rowland PW. A quantitative method for the estimation of clubbing. Scientific Session of the Senior Class, Tulane University Medical School 1961;11:299.

21. Waring WW, Wilkinson W, Wiebe RA, Faul BC, Hilman BC. Quantitation of digital clubbing in children. Am Rev Respir Dis $1971 ; 104: 166-74$.

22. Bentley D, Cline J. Estimation of clubbing by analysis of shadowgraph. Br Med J 1970;2:43.

23. Mellins RB, Fishman AP. Digital casts for study of clubbing of the fingers. Circulation 1966,33:143-5.

24. Neufeld 0, Wallbank WL. Clubbed fingers. Ohio Med J 1952;48:834-7.

25. Staven P. Instrument for estimation of clubbing. Lancet 1959;2:7-8.

26. Cudkowicz L, Wraith DG. An evaluation of the clinical significance of clubbing in common lung disorders. $\mathrm{Br} \mathrm{J}$ Tuberc 1957;51:14-31.

27. Pineda C. Diagnostic imaging in hypertrophic ostearthropathy. Clin Exp Rheumatol 1992;10(Suppl 7):27-33.

28. Rush PJ, Giorshev C, Shore A, Levinson H. The use of thermography in clubbing. Respir Med 1992;86:257-9.

29. Bigler C. The morphology of clubbing. Am J Pathol 1958; 34:237-61.

30. Lovell RRH. Observations on the structure of clubbed fingers. Clin Sci 1950;9:299-321.

31. Ward RW, Chin R Jr, Keyes JW Jr, Haponik EF. Digital clubbing demonstration with positron emission tomography. Chest 1995;107:1172-3.

32. Bentley D, Moore A, Swachman H. Finger clubbing. A quantitative survey by analysis of the shadowgraph. Lancet 1976;1:164-7.

33. Sinniah D, Omar A. Quantitation of digital clubbing by shadowgram technique. Arch Dis Child 1979;54:145-6.

34. Sly RM, Ghazanshai S, Buranakul B, Puapan P, Gupta S, Warren $\mathrm{R}$, et al. Objective assessment for digital clubbing in caucasian, negroe, and oriental subjects. Chest 1973;64:687-9.

35. Niewoehner DE, Kleinerman J, Rice DB. Pathologic changes in the peripheral airways of young cigarette smokers. N Engl J Med 1974;291:755-8.

36. Sadikot RT, Johnson J, Loyd JE, Christman JW. Respiratory bronchiolitis associated with severe dyspnea, exertional hypoxemia, and clubbing. Chest 2000;117:282-5. 